\title{
Mixed-integer second-order cone programming for lower hedging of American contingent claims in incomplete markets
}

\author{
Mustafa Ç. Pınar
}

Received: 14 May 2010 / Accepted: 22 August 2011 / Published online: 7 September 2011

(C) Springer-Verlag 2011

\begin{abstract}
We describe a challenging class of large mixed-integer second-order cone programming models which arise in computing the maximum price that a buyer is willing to disburse to acquire an American contingent claim in an incomplete financial market with no arbitrage opportunity. Taking the viewpoint of an investor who is willing to allow a controlled amount of risk by replacing the classical no-arbitrage assumption with a "no good-deal assumption" defined using an arbitrage-adjusted Sharpe ratio criterion we formulate the problem of computing the pricing and hedging of an American option in a financial market described by a multi-period, discrete-time, finite-state scenario tree as a large-scale mixed-integer conic optimization problem. We report computational results with off-the-shelf mixed-integer conic optimization software.
\end{abstract}

Keywords American options - Mixed-integer second-order cone optimization

\section{Introduction}

Hard discrete optimization problems and novel approaches to solve them have been an important part of research in optimization in the last decade; see, e.g., [19]. Mathematical finance offers a rich source of interesting optimization problems. The emergence of integer programming in mathematical finance has been so far limited mainly to portfolio optimization problems where buying and selling securities were subject to fixed transaction costs or when minimum lot restrictions were present. Cardinality constraints in the context of portfolio optimization may also lead to the use of discrete variables; see, e.g., [11,22].

Research supported by TUBITAK Grant 107K250.

M. Ç. Pınar ( $\varangle)$

Department of Industrial Engineering, Bilkent University, Ankara 06800, Turkey

e-mail: mustafap@bilkent.edu.tr 
In the present paper, we report on a different area of mathematical finance where finite dimensional optimization models with discrete-valued variables is of interest: pricing of stochastic cash flows. The pricing problem of stochastic cash flows is complicated by the fact that most financial markets are incomplete, i.e., not all future uncertain cash flows can be replicated exactly using the existing instruments. This observation leads to a wealth of literature on pricing and hedging in incomplete markets; see, e.g., $[4,6,9,21]$. When markets are incomplete state prices and claim prices are not unique. Since markets are almost never complete due to market imperfections as discussed in Carr et al. [6], and characterizing all possible future states of economy is impossible, the common practice is to find the cheapest portfolio dominating a stochastic future cash flow and the most expensive portfolio dominated by it, and use these respective values as bounds on the price of the stochastic cash flow. These bounds are referred to as super-replication and sub-replication bounds or no-arbitrage (or equilibrium) bounds. They are also known as upper hedging and the lower hedging prices. In the absence of arbitrage, the lower hedging price is the value of the most precious self-financing portfolio strategy composed of market instruments whose payoff is dominated by the contingent claim payoff at expiration. The lower hedging price can also be interpreted as the largest amount the contingent claim buyer can borrow (in the form of cash or by short-selling stocks) to acquire the claim while paying off his/her debt in a selffinanced manner using the contingent claim payoff at expiration [10]. Hence, we refer to this price as the buyer's price as well as the lower hedging price. For European contingent claims with no early exercise and termination possibility, the upper and lower hedging prices are expressed as supremum and infimum, respectively, of the expectation of the discounted contingent claim payoff (at expiration) over all probability measures that make the underlying stock price a martingale. We direct the reader to the book by Föllmer and Schied [14] for an in-depth treatment of pricing contingent claims in discrete time, and to Chalasani and Jha [10] for American contingent claims (ACC), which can be exercised at any time until expiration. The upper hedging price for ACC is the supremum of the expectation of the discounted contingent claim payoff (at some time between now and expiration) over all stopping times and all probability measures that make the underlying stock-price process a martingale. While the upper hedging price for ACC can be cast as a linear programming problem in discrete time $[10,20]$, the lower hedging price is harder to compute since it requires the solution of a mixed-integer programming problem [20]. Recently, Camc1 and Pinar [5] showed that the lower hedging price can also be computed by solving the LP relaxation of the mixed-integer model, i.e., the LP relaxation is exact. On the other hand, as indicated in [14], the computed upper and lower hedging prices may be far apart, and useless in practice. Furthermore, as we shall see in Sect. 6, the lower hedging bound may even turn out to be equal to zero!

In the present work, we are interested in pricing ACCs using a risk criterion introduced in [12] and further developed in [7,9]. Our work is based on good-deals defined as investments with high arbitrage adjusted Sharpe ratio [9]. Our motivation is to derive a higher (thus, more meaningful) lower hedging price for an ACC in an incomplete market. In a multi-period, discrete time, discrete state space framework we define the stock price process as a non-recombinant tree and formulate a mixed-integer second-order cone programming problem for computing the lower hedging price under the Sharpe 
ratio risk criterion (for recent work on second-order cone nonlinear programming, see [15]). After a brief introduction to the financial market setting, we describe our pricing measure and give our mixed-integer second-order cone optimization formulation. We develop an application using S\&P 500 index options data, and report our preliminary computational experience on these very difficult and large problem instances whose even convex relaxation may prove tough to handle. These instances clearly pose a challenge to the numerical optimization community. We obtain the optimal solution of the instances using duality theory.

The rest of the paper is organized as follows. In Sect. 2 we describe the financial market structure assumed in the paper. Section 3 introduces the Sharpe ratio risk criterion, and our initial formulation is given in Sect. 4. Section 5 describes the calibrated option bounds model used for numerical testing purposes. In Sect. 6 we present our numerical experiences with the instances generated using the calibrated option bounds setting, and our approach via duality to obtain an optimal solution to challenging instances. We conclude in Sect. 7.

\section{The stochastic scenario tree}

We approximate the behavior of the stock market by assuming that security prices and other payments are discrete random variables supported on a finite probability space $(\Omega, \mathcal{F}, P)$ whose atoms are sequences of real-valued vectors (asset values) over the discrete time periods $t=0,1, \ldots, T$ as in [16]. We further assume the market evolves as a discrete, non-recombinant scenario tree (hence, suitable for incomplete markets) in which the partition of probability atoms $\omega \in \Omega$ generated by matching path histories up to time $t$ corresponds one-to-one with nodes $n \in \mathcal{N}_{t}$ at level $t$ in the tree. The set $\mathcal{N}_{0}$ consists of the root node $n=0$, and the leaf nodes $n \in \mathcal{N}_{T}$ correspond one-to-one with the probability atoms $\omega \in \Omega$. The $\sigma$-algebras are such that, $\mathcal{F}_{0}=\{\emptyset, \Omega\}, \mathcal{F}_{t} \subset \mathcal{F}_{t+1}$ for all $0 \leq t \leq T-1$ and $\mathcal{F}_{T}=\mathcal{F}$. A stochastic process is said to be $\left(\mathcal{F}_{t}\right)_{t=0}^{T}$-adapted if for each $t=0, \ldots, T$, the outcome of the process only depends on which element of $\mathcal{F}_{t}$ has been realized at stage $t$. Similarly, a decision process is said to be $\left(\mathcal{F}_{t}\right)_{t=0}^{T}$-adapted if for each $t=0, \ldots, T$, the decision depends on which element of $\mathcal{F}_{t}$ has been realized at stage $t$. In the scenario tree, every node $n \in \mathcal{N}_{t}$ for $t=1, \ldots, T$ has a unique parent denoted $\pi(n) \in \mathcal{N}_{t-1}$, and every node $n \in \mathcal{N}_{t}, t=0,1, \ldots, T-1$ has a non-empty set of child nodes $\mathcal{C}(n) \subset \mathcal{N}_{t+1}$. We denote the set of all nodes in the tree by $\mathcal{N}$. We use the function $t(n)$ to hold the time period to which node $n$ belongs, i.e., $n \in \mathcal{N}_{t(n)}$. The symbol $\mathcal{A}(n)$ denotes the ascendant nodes or path history of node $n$ including itself, and $D(n)$ represents the descendant nodes from $n$ including itself. The probability distribution $P$ is obtained by attaching positive weights $p_{n}$ to each leaf node $n \in \mathcal{N}_{T}$ so that $\sum_{n \in \mathcal{N}_{T}} p_{n}=1$. For each non-terminal (intermediate level) node in the tree we have, recursively,

$$
p_{n}=\sum_{m \in \mathcal{C}(n)} p_{m}, \quad \forall n \in \mathcal{N}_{t}, t=T-1, \ldots, 0 .
$$

Hence, each intermediate node has a probability mass equal to the combined mass of the paths passing through it. 
Fig. 1 A sample scenario tree

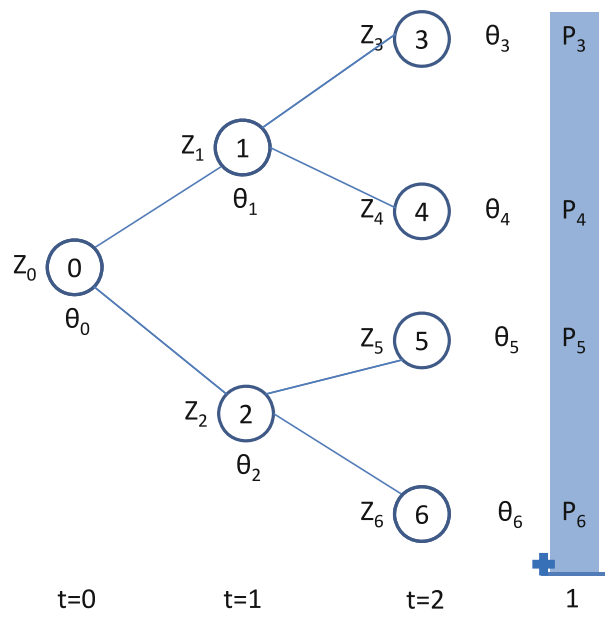

A random variable $X$ is a real valued function defined on $\Omega$. It can be lifted to the nodes of a partition $\mathcal{N}_{t}$ of $\Omega$ if each level set $\left\{X^{-1}(a): a \in \mathbb{R}\right\}$ is either the empty set or is a finite union of elements of the partition. In other words, $X$ can be lifted to $\mathcal{N}_{t}$ if it can be assigned a value on each node of $\mathcal{N}_{t}$ that is consistent with its definition on $\Omega$, [16]. This kind of random variable is said to be measurable with respect to the information contained in the nodes of $\mathcal{N}_{t}$. A stochastic process $\left\{X_{t}\right\}$ is a time-indexed collection of random variables such that each $X_{t}$ is measurable with respect $\mathcal{N}_{t}$. The expected value of $X_{t}$ is uniquely defined by the sum $\mathbb{E}^{P}\left[X_{t}\right]:=\sum_{n \in \mathcal{N}_{t}} p_{n} X_{n}$.

The market consists of $J+1$ traded securities indexed by $j=0,1, \ldots, J$ with prices at node $n$ given by the vector $Z_{n}=\left(Z_{n}^{0}, Z_{n}^{1}, \ldots, Z_{n}^{J}\right)$. We assume as in $[8,20]$ that the security indexed by 0 has strictly positive prices at each node of the scenario tree.

The amount of security $j$ held by the investor in state (node) $n \in \mathcal{N}_{t}$ is denoted $\theta_{n}^{j}$. Therefore, to each state $n \in \mathcal{N}_{t}$ is associated a vector $\theta_{n} \in \mathbb{R}^{J+1}$. The value of the portfolio at state $n$ is $Z_{n} \cdot \theta_{n}=\sum_{j=0}^{J} Z_{n}^{j} \theta_{n}^{j}$. A sample scenario tree for a market with three trading points is depicted in Fig. 1.

In our finite probability space setting an American contingent claim $H$ is a stochastic process measurable with respect to $N_{t}$, and hence, generates payoff opportunities $H_{n},(n \geq 0)$ to its holder depending on the states $n$ of the market.

The prevailing approach in the mathematical finance literature to the pricing of options is to find the fair price that does not allow arbitrage opportunities to the participants of the market. In an arbitrage-free financial market the lower hedging bound (or lower hedging price) is found by solving the following mixed-integer linear optimization problem NALHP (No-Arbitrage Lower Hedging Problem):

$$
\begin{aligned}
\max \quad-Z_{0} \cdot \theta_{0}+H_{0} e_{0} & \\
\text { s.t. } \quad Z_{n} \cdot\left(\theta_{n}-\theta_{\pi(n)}\right) & =e_{n} H_{n}, \quad \forall n \in \mathcal{N}_{t}, t \geq 1 \\
Z_{n} \cdot \theta_{n} & \geq 0, \quad \forall n \in \mathcal{N}_{T}, \\
\sum_{m \in \mathcal{A}(n)} e_{m} & \leq 1, \quad \forall n \in \mathcal{N}_{T} \\
e_{n} & \in\{0,1\}, \quad \forall n \in \mathcal{N} .
\end{aligned}
$$


where the optimal value gives the lower hedging price [5, 20]. However, as we shall see in Sect. 6, this model may yield unreasonable bounds (equal to zero), and may therefore be of limited use in practice. This situation can be remedied by the Sharpe-ratio based lower hedging bound of the present paper.

\section{Arbitrage-adjusted Sharpe ratio good-deals}

To motivate the good-deal opportunity in our context we shall use the following reasoning as in [7]. Let us relax the constraint that final wealth positions $Z_{n} \cdot \theta_{n}$ be non-negative for all $n \in \mathcal{N}_{T}$ by splitting every final wealth position $Z_{n} \cdot \theta_{n}$ into the sum of a non-negative component $v_{n}$ and an unrestricted-in-sign (free) component $x_{n}$ :

$$
Z_{n} \cdot \theta_{n}=v_{n}+x_{n} \quad \forall n \in \mathcal{N}_{T},
$$

and replacing the inequalities $Z_{n} \cdot \theta_{n} \geq 0, \forall n \in \mathcal{N}_{T}$ by Eq. (3.1). The above relaxation of the pricing problem might result in extremely large negative final wealth positions that are unacceptable for the person trying to construct a sequence of hedging portfolios for a given contingent claim. To limit the magnitude of negative final wealth positions, we impose the restriction that the expected value of free component $x_{n}$ of the final wealth remain non-negative and be at least a positive multiple of its standard deviation. In other words, if $x_{n}$, for $n \in \mathcal{N}_{T}$ represent the realizations of random variable $X$, using some positive real $\lambda$ we impose the restriction

$$
\mathbb{E}[X] \geq \lambda \sqrt{V[X]}
$$

where $\mathbb{E}[X]$ denotes expectation, $V[X]$ denotes variance of $X$. More precisely, in our setting this restriction translates into

$$
\sum_{n \in \mathcal{N}_{T}} p_{n} x_{n} \geq \lambda \sqrt{\sum_{n \in \mathcal{N}_{T}} p_{n}\left(x_{n}-\sum_{n \in \mathcal{N}_{T}} p_{n} x_{n}\right)^{2}} .
$$

We note that inequality (3.2) could be also be viewed as

$$
\frac{E[X]}{\sqrt{V[X]}} \geq \lambda
$$

which is precisely a lower-bound restriction on the Sharpe ratio [9] of the unrestricted-in-sign component of the final wealth position. We term the Sharpe ratio of the unrestricted-in-sign component of the final wealth position the "arbitrage-adjusted Sharpe ratio". Put in other words, we take the viewpoint of the investor who is willing to give up non-negative final wealth in all states of nature, and can accept some negative wealth positions provided that they satisfy restriction (3.3). The term "arbitrage-adjusted" reflects the feature that the new pricing problem introduced below tends to give identical results with no-arbitrage pricing problem (NAHLP) in the 
limit when $\lambda$ approaches infinity [7]. This development is motivated by the classical problem of finance where investors are interested in identifying zero cost investment opportunities with the highest possible Sharpe ratio. In our context, an investor who identifies a sequence of portfolio holdings resulting in non-negative wealth positions in all states of nature while hedging the cash outlays of a contingent claim can be thought of having achieved an infinite Sharpe ratio as follows. He/she can set the $x$ component of the final wealth position to zero in all states of nature while keeping the non-negative wealth positions in the variables $v_{n}$, which results in both zero expected value and zero variance of $x$, which may be combined with an infinitely large $\lambda$. Hence, we can identify a sequence of hedging portfolios for a contingent claim resulting in non-negative final wealth positions with an infinitely large arbitrage-adjusted Sharpe ratio.

Assume that there exist a set of vectors $\theta_{0}, \theta_{1}, \ldots, \theta_{|\mathcal{N}|}$ such that

$$
\begin{aligned}
Z_{0} \cdot \theta_{0} & =0 \\
Z_{n} \cdot\left(\theta_{n}-\theta_{\pi(n)}\right) & =0, \quad \forall n \in \mathcal{N}_{t}, t \geq 1
\end{aligned}
$$

and

$$
\sum_{n \in \mathcal{N}_{T}} p_{n} x_{n}-\lambda \sqrt{\sum_{n \in \mathcal{N}_{T}} p_{n}\left(x_{n}-\sum_{n \in \mathcal{N}_{T}} p_{n} x_{n}\right)^{2}}>0
$$

for $\lambda>0$. This sequence of portfolio holdings is said to yield a "Sharpe ratio gooddeal opportunity" at level $\lambda$. This formulation is similar to the Sharpe ratio criterion treated in $[9,12]$. Here, the parameter $\lambda$ can be interpreted as a loss aversion parameter of the individual investor because as $\lambda$ gets larger the investor is closer to seeking an arbitrage.

\section{The formulation}

Now, let us assume that an American contingent claim $H$ is available in our financial market setting. The potential buyer is interested in borrowing cash to acquire the claim, and with the remaining cash to form a portfolio of traded instruments. She/he will modify this portfolio later using proceeds from the claim (if exercised) or through self-financing transactions. At the expiry date of the option the final positions that the buyer carries should satisfy the Sharpe ratio risk constraint, i.e., the ratio of the average position to the standard deviation of the position should remain bounded by $\lambda$. The exercise of the American contingent claim is controlled by means of binary variables $e_{n}, n \in N$. Hence, under the assumption of no good-deal opportunities for the stock price process, the price of the ACC that provides no good-deals to the buyer must be greater than or equal to the optimal value of the following optimization problem (SP) (Sharpe Problem) 


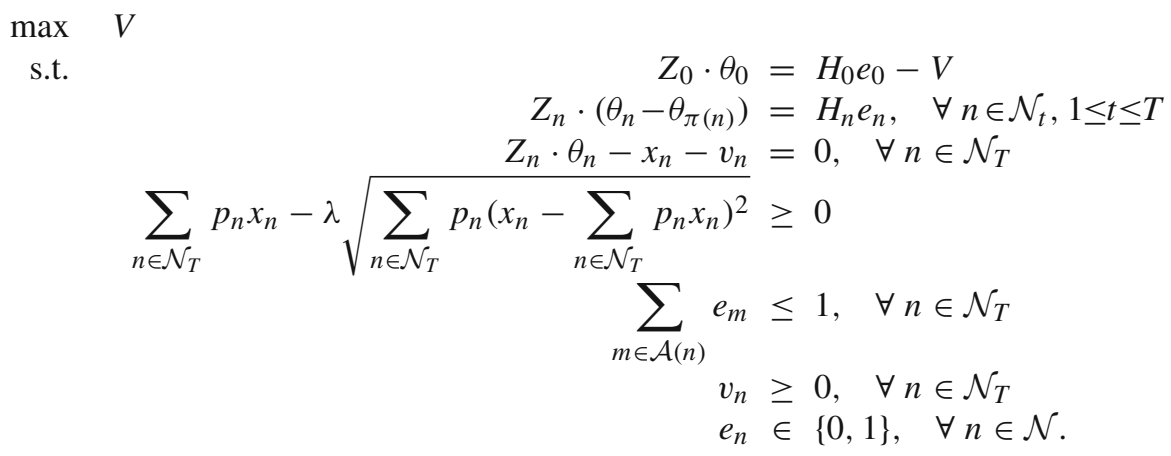

This problem is a mixed integer second-order cone programming (MISOCP) problem. The first constraint ties the initial wealth (borrowed) to the value of an initial portfolio. The second set of constraints represent the portfolio transactions at the nodes of subsequent periods. The third constraint is the Sharpe ratio good-deal constraint while the fourth set of constraints limits exercise to a single node over each sample path. This problem is less tightly constrained since every feasible solution to (NALHP) is a feasible solution for (SP) by taking $x_{n}=0$ for all $n$ and $v_{n}=Z_{n} \cdot \theta_{n}$. Therefore, we expect to obtain a lower hedging value at least as large as in (NALHP) by solving (SP). For reasons due to availability of data we shall work on a slightly different model described next.

\section{Calibrated option bounds}

In the setting of [17] adopted for our numerical tests, liquid European options traded in the market are used for hedging purposes in addition to securities. These liquid options give the investor the possibility of forming buy-and-hold strategies in the hedging portfolio sequence. That is, every liquid option can be bought or shorted by the investor at time zero with the purpose of hedging a contingent claim, and no intermediary trading is available for these options, i.e., once a position (long or short) is taken in an option, this position is kept fixed until maturity of the option. Assuming there are $M$ such liquid options, we denote them by $G^{k}, k=1, \ldots, M$. Bid and ask prices observed in the market at time 0 for option $k$ are denoted by $C_{b}^{k}$ and $C_{a}^{k}$, respectively, with the latter greater than or equal to the former. $G_{n}^{k}$ is the payoff of option $k$ at node $n$ of the scenario three and $G_{n}$ is the vector of option payoffs at node $n$. At this point we will assume that $S_{n}^{0}=1, \forall n \in \mathcal{N}$, to use normal stock prices instead of discounted prices. This assumption is consistent with our numerical experiments since we use a zero interest rate. The non-negative $M$-vectors $\xi_{+}$and $\xi_{-}$denote, respectively, the long and short initial buy-and-hold positions in the liquid options. Under these assumptions the buyer's problem we shall be solving is modified as [referred to as (P)] 


$$
\begin{aligned}
& \max \quad-Z_{0} \cdot \theta_{0}-C_{a} \cdot \xi_{+}+C_{b} \cdot \xi_{-}+H_{0} e_{0} \\
& \text { s.t. } \quad Z_{n} \cdot\left(\theta_{n}-\theta_{\pi(n)}\right)=G_{n} \cdot\left(\xi_{+}-\xi_{-}\right)+e_{n} H_{n}, \quad \forall n \in \mathcal{N}_{t}, t \geq 1 \\
& \sum_{n \in \mathcal{N}_{T}} p_{n} x_{n}-\lambda \sqrt{\sum_{n \in \mathcal{N}_{T}} p_{n}\left(x_{n}-\sum_{n \in \mathcal{N}_{T}} p_{n} x_{n}\right)^{2}} \geq 0 \\
& \begin{aligned}
v_{n}+x_{n} & =Z_{n} \cdot \theta_{n}, \quad \forall n \in \mathcal{N}_{T}, \\
\sum_{m \in \mathcal{A}(n)}^{e_{m}} & \leq 1, \quad \forall n \in \mathcal{N}_{T}
\end{aligned} \\
& e_{n} \in\{0,1\}, \quad \forall n \in \mathcal{N} \\
& \xi_{+}, \xi_{-} \geq 0, \\
& v_{n} \geq 0, \quad \forall n \in \mathcal{N}_{T} \text {. }
\end{aligned}
$$

\section{Numerical results and solution via duality}

We use 48 European options written on the S\&P 500 index. The option data were available in the market on 10 September 2002. The first 21 are call options and the remaining ones are put options. Strikes and maturities as well as actual bid and ask prices (columns $C_{b}$ and $C_{a}$ ) of these options are given in Table 1. We compute "calibrated" pricing bounds for each option treating that option as an American option. This means that the buyer or writer of the option can include buy-andhold positions in the 47 remaining European options into his/her hedge portfolio sequence.

We use $Z=\left(1, Z^{1}\right)$ as the traded securities. Having $Z^{0}=1$ for all dates means that interest rate is zero. We assume that the price of the S\&P 500 index (i.e., $Z^{1}$ ) follows a geometric Brownian motion. Under this assumption, we generate a scenario tree by the Gauss-Hermite process which was discussed in $[17,18]$ in detail. We use a branching structure of $(50,10,10)$. It means that the tree divides into 50 nodes at the second period. Then, each node branches into ten nodes in the second period hence there are additional 500 nodes in the third period. Then, again each node of the third period is divided into 10 and there are 5,000 leaf nodes of the tree. We assume that investors can trade at days 0, 17, 37 and 100, and form instances of problem (P) with four periods, which already yields huge MISOCP instances with up to 25,553 constraints and 31,749 variables of which 5,551 are binary. Let $K$ denote the strike price. We have now $H_{n}=\left(Z_{n}^{1}-K\right)_{+}$for call options and $H_{n}=\left(K-Z_{n}^{1}\right)_{+}$for put options for all $n \in N$.

We used the conic interior point optimizer MOSEK version 5.0.0.127 [2] under default settings through GAMS version 23.2 [1] to determine buyer's prices for each option for $\lambda=5.7$, a value that was chosen because it leads to price bounds that are closer to reported bid and ask prices for all the options and also leads to improved price bounds in most cases (see Table 2). We report computational results in Table 1. It proved impossible to get most MINLP solvers to work on the present instances using the primal formulation (P). MOSEK was the only code to solve the convex relaxations with some success, but it stopped immediately with an error message when the models were input as mixed-integer conic models. In Table 1 below, the continuous (relaxed) models marked with a B for "binary" solution, and solver and model status both 
Table 1 Numerical results for the continuous relaxation of Sharpe ratio lower hedging primal problem (P) with $\lambda=5.7$

\begin{tabular}{|c|c|c|c|c|c|c|c|c|}
\hline Option no. & Type & Strike & Maturity & $C_{b}$ & $C_{a}$ & Buyer's price & Solver \& model status & Solution \\
\hline 1 & Call & 890 & 17 & 31.5 & 33.5 & 31.22 & 1,1 & B \\
\hline 2 & Call & 900 & 17 & 24.4 & 26.4 & 24.67 & 1,1 & B \\
\hline 3 & Call & 905 & 17 & 21.2 & 23.2 & 21.80 & 1,1 & B \\
\hline 4 & Call & 910 & 17 & 18.5 & 20.1 & 18.92 & 1,1 & B \\
\hline 5 & Call & 915 & 17 & 15.8 & 17.4 & 16.09 & 1,1 & B \\
\hline 6 & Call & 925 & 17 & 11.2 & 12.6 & - & 4,6 & - \\
\hline 7 & Call & 935 & 17 & 7.6 & 8.6 & 7.68 & 1,1 & B \\
\hline 8 & Call & 950 & 17 & 3.8 & 4.6 & 3.39 & 1,1 & B \\
\hline 9 & Call & 955 & 17 & 3 & 3.7 & 2.99 & 1,1 & B \\
\hline 10 & Call & 975 & 17 & 0.95 & 1.45 & 0.65 & 1,1 & B \\
\hline 11 & Call & 980 & 17 & 0.65 & 1.15 & 0.66 & 1,1 & B \\
\hline 12 & Call & 900 & 37 & 42.3 & 44.3 & 40.58 & 1,1 & $\mathrm{~F}$ \\
\hline 13 & Call & 925 & 37 & 28.2 & 29.6 & - & 4,6 & - \\
\hline 14 & Call & 950 & 37 & 17.5 & 19 & - & 4,6 & - \\
\hline 15 & Call & 875 & 100 & 77.1 & 79.1 & 75.49 & 1,1 & F \\
\hline 16 & Call & 900 & 100 & 61.6 & 63.6 & 59.87 & 1,1 & $\mathrm{~F}$ \\
\hline 17 & Call & 950 & 100 & 35.8 & 37.8 & - & 4,6 & - \\
\hline 18 & Call & 975 & 100 & 26 & 28 & - & 4,6 & - \\
\hline 19 & Call & 995 & 100 & 19.9 & 21.5 & - & 4,6 & - \\
\hline 20 & Call & 1,025 & 100 & 12.6 & 14.2 & - & 4,6 & - \\
\hline 21 & Call & 1,100 & 100 & 3.4 & 3.8 & - & 4,6 & - \\
\hline 22 & Put & 750 & 17 & 0.4 & 0.6 & - & 4,6 & - \\
\hline 23 & Put & 790 & 17 & 1 & 1.3 & 1.01 & 1,1 & B \\
\hline 24 & Put & 800 & 17 & 1.3 & 1.65 & 1.21 & 1,1 & B \\
\hline 25 & Put & 825 & 17 & 2.5 & 2.85 & 2.05 & 1,1 & B \\
\hline 26 & Put & 830 & 17 & 2.6 & 3.1 & 2.74 & 1,1 & B \\
\hline 27 & Put & 840 & 17 & 3.4 & 3.8 & 3.41 & 4,6 & - \\
\hline 28 & Put & 850 & 17 & 3.9 & 4.7 & 4.41 & 1,1 & B \\
\hline 29 & Put & 860 & 17 & 5.5 & 5.8 & 5.39 & 4,6 & - \\
\hline 30 & Put & 875 & 17 & 7.2 & 7.8 & 7.60 & 4,6 & - \\
\hline 31 & Put & 885 & 17 & 9.4 & 10.4 & 10.40 & 1,1 & B \\
\hline 32 & Put & 750 & 37 & 5.5 & 5.9 & - & 2,6 & - \\
\hline 33 & Put & 775 & 37 & 6.9 & 7.7 & 7.60 & 1,1 & $\mathrm{~F}$ \\
\hline 34 & Put & 800 & 37 & 9.3 & 10 & 9.89 & 1,1 & $\mathrm{~F}$ \\
\hline 35 & Put & 850 & 37 & 16.7 & 18.3 & - & 4,6 & - \\
\hline 36 & Put & 875 & 37 & 23 & 24.3 & 22.39 & 1,1 & $\mathrm{~F}$ \\
\hline 37 & Put & 900 & 37 & 31 & 33 & 32.70 & 1,1 & F \\
\hline 38 & Put & 925 & 37 & 41.8 & 43.8 & 43.61 & 1,1 & $\mathrm{~F}$ \\
\hline 39 & Put & 975 & 37 & 73 & 75 & 72.57 & 1,1 & $\mathrm{~F}$ \\
\hline 40 & Put & 995 & 37 & 88.9 & 90.9 & - & 4,6 & - \\
\hline
\end{tabular}


Table 1 continued

\begin{tabular}{lllllllll}
\hline Option no. & Type & Strike & Maturity & \multicolumn{1}{c}{$C_{b}$} & \multicolumn{1}{c}{$C_{a}$} & Buyer's price & Solver \& model status & Solution \\
\hline 41 & Put & 650 & 100 & 5.7 & 6.7 & 2.73 & 1,1 & $\mathrm{~F}$ \\
42 & Put & 700 & 100 & 9.2 & 10.2 & 10.10 & 1,1 & $\mathrm{~F}$ \\
43 & Put & 750 & 100 & 14.7 & 15.8 & - & 4,6 & - \\
44 & Put & 775 & 100 & 17.6 & 19.2 & - & 4,6 & - \\
45 & Put & 800 & 100 & 21.7 & 23.7 & - & 4,6 & - \\
46 & Put & 850 & 100 & 33.3 & 35.3 & 32.93 & 1,1 & $\mathrm{~F}$ \\
47 & Put & 875 & 100 & 40.9 & 42.9 & - & 4,6 & - \\
48 & Put & 900 & 100 & 50.3 & 52.3 & 51.99 & 1,1 & $\mathrm{~F}$ \\
\hline
\end{tabular}

Solver Status Codes: 1, Normal Completion; 2, Iteration Interrupt; 4, Terminated by Solver Model Status Codes: 1, Optimal; 6, Intermediate Infeasible; F, Fractional; B, Binary

equal to $1^{1}$ were solved successfully with MOSEK, and yielded an integer $e$ component hence the optimal solution to the original model for claims with the earliest maturity date (day 17). However, for the remaining models either the solver successfully returned an optimal fractional solution (hence, an upper bound on the buyer's price), or stopped with an error message (reported in the table). It is conjectured that the solvers run into numerical problems due to the very small entries for the node probabilities generated from the Gauss-Hermite process. For models with five trading dates that are even larger, it is expected that the problems will grow even more challenging.

The key to turn this situation around is via duality. Before we can carry on, we need the following definition (see [16] for details).

Definition 1 If there exists a probability measure $Q=\left\{q_{n}\right\}_{n \in \mathcal{N}_{T}}$ such that

$$
Z_{t}=\mathbb{E}^{q}\left[Z_{t+1} \mid \mathcal{N}_{t}\right](t \leq T-1),
$$

i.e.,

$$
\begin{gathered}
q_{m} Z_{m}=\sum_{n \in \mathcal{C}(m)} q_{n} Z_{n}, \quad \forall m \in \mathcal{N}_{t}, 0 \leq t \leq T-1 \\
q_{0}=1, \\
q_{n} \geq 0, \quad \forall n \in \mathcal{N},
\end{gathered}
$$

then the vector process $\left\{Z_{t}\right\}$ is called a vector-valued martingale under $Q$, and $Q$ is called a martingale probability measure for the process.

Let $E$ be the set $\left\{e_{n} \mid \sum_{m \in \mathcal{A}(n)} e_{m} \leq 1, \forall n \in \mathcal{N}_{T}\right.$ and $\left.e_{n} \in\{0,1\}, \forall n \in \mathcal{N}\right\}$. Consider problem (P) for fixed $e \in E$. Based on our earlier work [7], for fixed $e \in E$

\footnotetext{
1 The Solver Status refers to the state (e.g., Normal completion, iteration or CPU limit interrupt, or some other termination status) of the solver program (e.g., CPLEX or MOSEK) on exit, and the Model Status gives a description of what the solution looks like (e.g., whether the solution reported is optimal, or locally optimal, or infeasible etc.)
} 
Table 2 Numerical results on the most difficult instances of Table 1 for Sharpe ratio lower hedging bounding problems (DL) and (DU) with $\lambda=5.7$

\begin{tabular}{|c|c|c|c|c|c|c|}
\hline Option no. & Type & Strike & Maturity & UB & LB & LHB \\
\hline 6 & Call & 925 & 17 & 10.57 & 10.57 & 10.42 \\
\hline 12 & Call & 900 & 37 & 40.58 & 40.58 & 40.58 \\
\hline 13 & Call & 925 & 37 & 26.86 & 26.86 & 26.86 \\
\hline 14 & Call & 950 & 37 & 14.32 & 14.32 & 13.82 \\
\hline 15 & Call & 875 & 100 & 75.48 & 75.48 & 75.48 \\
\hline 16 & Call & 900 & 100 & 59.88 & 59.88 & 59.88 \\
\hline 17 & Call & 950 & 100 & 33.81 & 33.80 & 32.27 \\
\hline 18 & Call & 975 & 100 & 24.99 & 24.99 & 23.70 \\
\hline 19 & Call & 995 & 100 & 18.90 & 18.90 & 17.72 \\
\hline 20 & Call & 1,025 & 100 & 10.06 & 10.03 & 8.01 \\
\hline 21 & Call & 1,100 & 100 & 0.389 & 0.384 & 0 \\
\hline 22 & Put & 750 & 17 & 0.545 & 0.545 & 0 \\
\hline 27 & Put & 840 & 17 & 3.44 & 3.44 & 3.20 \\
\hline 29 & Put & 860 & 17 & 5.41 & 5.41 & 4.60 \\
\hline 30 & Put & 875 & 17 & 7.65 & 7.65 & 6.80 \\
\hline 32 & Put & 750 & 37 & 3.80 & 3.80 & 3.80 \\
\hline 33 & Put & 775 & 37 & 7.60 & 7.60 & 6.32 \\
\hline 34 & Put & 800 & 37 & 9.89 & 9.89 & 7.90 \\
\hline 35 & Put & 850 & 37 & 15.84 & 15.84 & 13.48 \\
\hline 36 & Put & 875 & 37 & 22.43 & 22.43 & 21.77 \\
\hline 37 & Put & 900 & 37 & 32.72 & 32.72 & 32.72 \\
\hline 38 & Put & 925 & 37 & 43.62 & 43.62 & 43.62 \\
\hline 39 & Put & 975 & 37 & 72.70 & 72.70 & 72.23 \\
\hline 40 & Put & 995 & 37 & 87.72 & 87.72 & 87.08 \\
\hline 41 & Put & 650 & 100 & 2.72 & 2.72 & 2.60 \\
\hline 42 & Put & 700 & 100 & 10.02 & 10.02 & 6.65 \\
\hline 43 & Put & 750 & 100 & 15.02 & 15.02 & 11.79 \\
\hline 44 & Put & 775 & 100 & 17.84 & 17.84 & 16.95 \\
\hline 45 & Put & 800 & 100 & 21.04 & 21.03 & 20.06 \\
\hline 46 & Put & 850 & 100 & 32.97 & 32.97 & 32.74 \\
\hline 47 & Put & 875 & 100 & 42.52 & 42.52 & 42.52 \\
\hline 48 & Put & 900 & 100 & 52.02 & 52.02 & 52.02 \\
\hline
\end{tabular}

the maximization over the remaining variables admits the dual problem (DL) below over the variables $q_{n}$. The primal problem (P) with fixed variables $e_{n}$ and the dual problem (DL) below have a common optimal value under a strict feasibility assumption to guarantee zero duality gap: ${ }^{2}$

\footnotetext{
2 There exists feasible $\theta, x, v$ such that the conic constraint in problem SP is satisfied as a strict inequality, and there exists feasible $q$ in DL such that constraint (6.5) holds as a strict inequality, see [3].
} 


$$
\min \sum_{n \in N} e_{n} q_{n} H_{n}
$$

subject to (6.1)-(6.3), (6.5), (6.6) as defined below:

$$
\begin{gathered}
\sqrt{\sum_{n \in \mathcal{N}_{T}} p_{n}\left(\frac{q_{n}}{p_{n}}-1\right)^{2}} \leq \lambda, \\
C_{b} \leq \sum_{t=1}^{T} \sum_{n \in N_{t}} q_{n} G_{n} \leq C_{a} .
\end{gathered}
$$

Now, defining $\tilde{\mathbf{Q}}$ to be set of all martingale measures satisfying the side conditions (6.5), (6.6), i.e., all vectors with components $\left\{q_{n}\right\}$ satisfying (6.1)-(6.3), (6.5), (6.6), we can express problem $\mathrm{P}$ as the computation of

$$
V_{0} \equiv \max _{e \in E} \min _{q \in \tilde{\mathbf{Q}}} \mathbb{E}^{q}\left[\sum_{t=0}^{T} H_{t} e_{t}\right] \equiv \sum_{n \in N} e_{n} q_{n} H_{n},
$$

where $V_{0}$ denotes the lower hedging value at node 0 , i.e., at $t=0$.

We denote by $V_{n}$ the value of the index option at node $n$ of the scenario tree at time $t(n)$. Let us define

$E_{n}=\left\{e_{\ell} \mid \sum_{m \in \mathcal{A}(\tilde{n}) \cap D(n)} e_{m} \leq 1, \quad \forall \tilde{n} \in \mathcal{N}_{T} \cap D(n) \quad\right.$ and $\left.\quad e_{\ell} \in\{0,1\}, \quad \forall \ell \in \mathcal{D}(n)\right\}$ as the set of exercise constraints for the sub-tree starting at node $n$. Likewise we define $\tilde{\mathbf{Q}}_{n}$ be the set of $q_{\ell} \geq 0$ for $\ell \in D(n)$ satisfying

$$
\begin{aligned}
& q_{\ell} Z_{\ell}=\sum_{m \in \mathcal{C}(\ell)} q_{m} Z_{m}, \quad \forall \ell \in \mathcal{N}_{t} \cap\left(D(n) \backslash\left(D(n) \cap N_{T}\right)\right), t(n) \leq t \leq T-1 \\
& q_{n}=1, \\
& \\
& \sqrt{\sum_{\ell \in \mathcal{N}_{T} \cap D(n)} p_{\ell}\left(\frac{q_{\ell}}{p_{\ell}}-1\right)^{2}} \leq \lambda, \\
& \tilde{C}_{b}^{n} \leq \sum_{t=t(n)+1}^{T} \sum_{\ell \in N_{t} \cap D(n)} q_{\ell} G_{\ell} \leq \tilde{C}_{a}^{n},
\end{aligned}
$$

where $\tilde{C}_{b}^{n}$ and $\tilde{C}_{a}^{n}$ are the bid and ask prices for the liquid options available for buyand-hold strategy at time point $t=t(n)$ and node $n$. Using the above definitions, the value $V_{n}$ of the index option at node $n$ is given as the solution to the problem 


$$
V_{n} \equiv \max _{e \in E_{n}} \min _{q \in \tilde{\mathbf{Q}}_{n}} \mathbb{E}^{q}\left[\sum_{t=t(n)}^{T} H_{t} e_{t}\right] .
$$

As an example, consider the scenario tree in Fig. 1. Choosing node 1, i.e., $n=1$, the set $\tilde{\mathbf{Q}}_{1}$ is the set of non-negative $q_{1}, q_{3}, q_{4}$ with $q_{1}=1$ such that

$$
\begin{gathered}
1=q_{3}+q_{4}, \\
Z_{1}^{1}=Z_{3}^{1} q_{3}+Z_{4}^{1} q_{4}, \\
\sqrt{p_{3}\left(q_{3} / p_{3}-1\right)^{2}+p_{4}\left(q_{4} / p_{4}-1\right)^{2}} \leq 1,
\end{gathered}
$$

and

$$
\tilde{C}_{b}^{1} \leq q_{3} G_{3}+q_{4} G_{4} \leq \tilde{C}_{a}^{1}
$$

where $\tilde{C}_{b}^{1}$ and $\tilde{C}_{a}^{1}$ are the bid and ask prices for the liquid options available for purchase/short sale at time point $t=1$ and node $n=1$. Now, we can prove the following result.

Theorem 1 For problem $(P)$, early exercise (before maturity $T$ ) is never strictly optimal, i.e., there always exists an optimal solution where the optimal values of exercise variables are $e_{n}^{*}=1$ for all $n \in N_{T}$.

Proof To prove the assertion we shall compare the value $V_{n}$ at an arbitrary intermediate node $n$ (i.e., $t(n)<T$ ) of the scenario tree to the call option payoff $Z_{n}-X$ at that node:

$$
\begin{aligned}
V_{n} & =\max _{e \in E_{n}} \min _{q \in \tilde{\mathbf{Q}}_{n}} \mathbb{E}^{q}\left[\sum_{t=t(n)}^{T} H_{t} e_{t}\right] \\
& =\max _{e \in E_{n}} \min _{q \in \tilde{\mathbf{Q}}_{n}} \mathbb{E}^{q}\left[\sum_{t=t(n)}^{T} e_{t}\left(Z_{t}-X\right)_{+}\right] \\
& =\max _{e \in E_{n}} \mathbb{E}^{q^{*}}\left[\sum_{t=t(n)}^{T} e_{t}\left(Z_{t}-X\right)_{+}\right] \\
& \geq \mathbb{E}^{q^{*}}\left[\left(Z_{T}-X\right)_{+}\right] \\
& \geq \mathbb{E}^{q^{*}}\left[Z_{T}-X\right] \\
& =Z_{t}-X,
\end{aligned}
$$

where the last equality follows since the price process $Z_{t}$ is a martingale under any member of $\tilde{\mathbf{Q}}_{n}$ (we suppressed the possible dependence of $q^{*}$ on $e$ to avoid further notational confusion). This chain of equations and inequalities proves that the option's intrinsic value $V_{n}$ at any node $n$ is at least as high as the benefit from immediate exercise, hence, exercise can be delayed without incurring a loss. Therefore, even 
if it is optimal to early exercise the option there must exist another optimal solution where exercise at the leaf nodes is optimal. The proof is identical for a put option with payoff $X-Z$ after the necessary changes.

Remark 1 The above result is valid under the condition that the riskless asset, $Z^{0}$, has price equal to one at all time periods and all nodes of the scenario tree, which is equivalent to saying that the risk-free interest rate is zero. If this condition is not satisfied, but $Z^{0}$ has strictly positive prices at all nodes, representations for the value of the option similar to (6.7) and (6.12) can be shown, after normalizing all stock prices and option payoffs at all nodes by dividing the stock price by the riskless security price, thereby introducing a discount factor $\beta_{t} \in(0,1]$ (with components $\beta_{n}$ for all $n \in N_{t}$ ) for all time periods $t=1, \ldots, T$. So, one would obtain the representation

$$
V_{0} \equiv \max _{e \in E} \min _{q \in \tilde{\mathbf{Q}}} \mathbb{E}^{q}\left[\sum_{t=0}^{T} \beta_{t} H_{t} e_{t}\right] \equiv \sum_{n \in N} \beta_{n} e_{n} q_{n} H_{n} .
$$

However, the proof of Theorem 1 breaks down in this case, and, indeed early exercise may become strictly optimal.

On the other hand, the result does not hold true for ACC with an arbitrary payoff structure $H_{n}$ even with zero riskless interest rate.

Solving the problem (DL) with $e$ fixed gives a lower bound to the optimal value $O P T(P)$. Using the above result we fix $e_{n}=1$ for all $n \in N_{T}$ (and thereby force all other $e$ 's to zero) and solve the above problem (DL) successfully using GAMS/MOSEK (solver returns an optimal solution with default parameter settings). The results are reported in Table 2 under column "LB" for lower bound.

As a verification we also solve the dual of the continuous relaxation of $(\mathrm{P})$ to give an upper bound. This dual problem, referred to as (DU), is the following problem (after some algebra and elimination of variables, the details of which are left to the reader):

$$
\min \sum_{n \in N_{T}} q_{n} H_{n}
$$

subject to (6.1)-(6.3), (6.5), (6.6), (6.15) where (6.15) is as defined below:

$$
q_{n} H_{n} \leq \sum_{m \in C(n)} q_{m} H_{m} \quad \forall n \in N_{t}, 0 \leq t \leq T-1 .
$$

Solving problem (DU) successfully (solver returned optimal solution with default settings) in GAMS/MOSEK we find that the lower and upper bounds collapse for almost all the difficult instances, as expected. The results are reported in Table 2 . The small discrepancies (always after the decimal point) between upper bounds of Table 1 and those of Table 2 are attributed to the numerical difficulties experienced by the conic solver when faced with the primal problem that is deemed less stable compared to the dual models. 
In Table 2 we also report in the last column under "LHB" (for lower hedging bound) the no-arbitrage lower hedging price obtained for the given option by solving the problem (CNALHP) (calibrated no-arbitrage lower hedging problem):

$$
\begin{aligned}
\max \quad-Z_{0} \cdot \theta_{0}-C_{a} \cdot \xi_{+} & +C_{b} \cdot \xi_{-}+H_{0} e_{0} \\
\text { s.t. } \quad Z_{n} \cdot\left(\theta_{n}-\theta_{\pi(n)}\right) & =G_{n} \cdot\left(\xi_{+}-\xi_{-}\right)+e_{n} H_{n}, \quad \forall n \in \mathcal{N}_{t}, t \geq 1 \\
Z_{n} \cdot \theta_{n} & \geq 0, \quad \forall n \in \mathcal{N}_{T}, \\
\sum_{m \in \mathcal{A}(n)} e_{m} & \leq 1, \quad \forall n \in \mathcal{N}_{T} \\
e_{n} & \in\{0,1\}, \quad \forall n \in \mathcal{N} \\
\xi_{+}, \xi_{-} & \geq 0 .
\end{aligned}
$$

Notice that (CNALHP) is a mixed-integer linear optimization problem which is solved easily by GAMS/CPLEX version 12.1 mixed-integer linear programming solver [13]. Incidentally, a result similar to Theorem 1 holds in this case too, but since GAMS/CPLEX easily handles our instances we do not need to make use of this property here.

As expected, in some options the lower hedging bound obtained using the Sharperatio based criterion is significantly higher than the no-arbitrage lower hedging bound which can even be equal to zero (option nos. 21 and 22)! Therefore, the Sharpe-ratio based lower hedging bound indeed delivers in some cases a more reasonable price bound in comparison with the no-arbitrage bound.

\section{Conclusions}

We presented a mixed-integer second-order cone programming formulation to compute a lower hedging price under a Sharpe ratio risk criterion for ACC in incomplete financial markets. The lower hedging price computed under the no-arbitrage criterion can be too low (even zero) in these markets while the Sharpe ration criterion remedies this problem. We constructed very challenging, large MISOCP instances that cannot be solved by state-of-the-art software. We managed to obtain an optimal solution to these instances using duality theory and a structural property of the optimal solution. Nonetheless, the instances remain a challenge for the numerical optimization developers.

\section{References}

1. Brooke, A., Kendrick, B., Meeraus, A.: GAMS: A User's Guide. The Scientific Press, San Fransisco (1992)

2. Mosek ApS. Mosek solver manual. Technical report, Mosek ApS c/o Symbion Science Park, Fruebjergvej 3, Box 15, 2100 Copenhagen, Denmark (2009)

3. Ben-Tal, A., Nemirovski, A.: Lectures on Modern Convex Optimization: Models, Algorithms and Applications. SIAM, Philadelphia (2001)

4. Bernardo, A.E., Ledoit, O.: Gain, loss and asset pricing. J. Polit. Econ. 108, 144-172 (2000) 
5. Camcı, A., Pınar, M. Ç.: Pricing American contingent claims by stochastic linear programming. Optimization 58, 627-640 (2009)

6. Carr, P., Geman, H., Madan, D.B.: Pricing and hedging in incomplete markets. J. Financ. Econ. 62, 131167 (2001)

7. Pınar, M.Ç.: Sharpe-ratio pricing and hedging of contingent claims in incomplete markets by convex programming. Automatica 44, 2063-2073 (2008)

8. Pınar, M.Ç., Altay-Salih, A., Camcı, A.: Expected gain-loss pricing and hedging of contingent claims in incomplete markets by linear programming. Eur. J. Oper. Res. 201, 770-785 (2010)

9. Cerny, A.: Generalized Sharpe ratios and pricing in incomplete markets. Eur. Financ. Rev. 7, 191233 (2003)

10. Chalasani, P., Jha, S.: Randomized stopping times and american option pricing with transaction costs. Math. Financ. 11, 33-77 (2001)

11. Chang, T.J., Meade, N., Beasley, J.E., Sharaiha, Y.M.: Heuristics for cardinality constrained portfolio optimization. Comput. Oper. Res. 27, 1271-1302 (2000)

12. Cochrane, J.H., Saa-Requejo, J.: Beyond arbitrage: Good-deal asset price bounds in incomplete markets. J. Polit. Econ. 108, 79-119 (2000)

13. CPLEX. Cplex 12 solver manual. Technical Report, IBM ILOG (2010)

14. Föllmer, H., Schied, A.: Stochastic Finance: An Introduction in Discrete Time. W. De Gruyter, Berlin (2004)

15. Kato, H., Fukushima, M.: An sqp-type algorithm for nonlinear second-order cone programs. Optim. Lett. 1, 129-144 (2007)

16. King, A.J.: Duality and martingales: a stochastic programming perspective on contingent claims. Math. Program. Ser. B 91, 543-562 (2002)

17. King, A.J., Koivu, M., Pennanen, T.: Calibrated option bounds. Int. J. Theor. Appl. Financ. 8, 141-159 (2005)

18. Omberg, E.: Efficient discrete time jump process models in option pricing. J. Financ. Quant. Anal. 23, 161-164 (1988)

19. Pardalos, P.M., Wolkowicz, H.: Novel Approaches to Hard Discrete Optimization. American Mathematical Society, USA (2003)

20. Pennanen, T., King, A.: Arbitrage pricing of American contingent claims in incomplete markets - a convex optimization approach. Technical Report, Helsinki School of Economics (2006)

21. Staum, J.: Fundamental theorems of asset pricing for good deal bounds. Math. Financ. 14, 141-161 (2004)

22. Zenios, S.A. (eds.): Financial Optimization. Cambridge University Press, Cambridge (1993) 